\title{
Development of a Neuron Based Internet Game Driven by a Brain-Computer Interface System
}

\author{
Unjoo Lee ${ }^{1}$, Seung Hoon Han ${ }^{1}$, Han Sup Kim ${ }^{1}$, Young Bum Kim ${ }^{1}$, Hyun Gi Jung ${ }^{1}$, Hyun-joo \\ $\mathrm{Lee}^{2}$, Yiran Lang ${ }^{2}$, Daehwan Kim ${ }^{2}$, Meiying Jin ${ }^{2}$, Jungwha Song ${ }^{1}$, Sungho Song ${ }^{1}$, Chang Geun \\ Song $^{1}$, and Hyung-Cheul Shin ${ }^{2}$ \\ ${ }^{1}$ Division of Information Engineering and Telecommunications, Hallym University \\ ${ }^{2}$ College of Medicine, Hallym University \\ hcshin@hallym.ac.kr
}

\begin{abstract}
Have you ever imagined that you could play an internet game against an animal like a rat? How would you feel if you lose? In the present study, we have developed an internet game driven by and linked to a brain-computer interface (BCI) system. The BCI system translated real-time neuronal activities from prefrontal cortex (PFC), or hippocampaus (CA1) of a rat into external device control commands and used them to drive a neuron based internet game series called RaviDuel.
\end{abstract}

\section{Introduction}

A brain-computer interface (BCI) is a communication channel which transforms a subject's thought processes into command signals to control various devices, for example, a computer application, a wheelchair, a robot arm, or a neural prosthesis. Many studies have been made on the prediction of human voluntary movement intention in real-time based on invasive or noninvasive methods to help severely motor-disabled persons by offering some abilities of motor controls and communications. Noninvasive method records electroencephalographic (EEG) signals and extracts intentional traits or movement-related EEG features, such as the P300 component of an eventrelated evoked potential (ERP) [1], EEG mu rhythm conditioning [2 4], or visually evoked potential (VEP) [5]. Noninvasive method has low spatial resolution since it takes readings from the entire brain rather than a specific part of the brain [6]. On the other hand, invasive method delivers better signal quality at the expense of its invasive characteristic. Its typical approaches include electrocorticograms [7], single neuron recordings [8], or multi-neuron population recordings [9]. Advanced researches on invasive methods are being actively pursued with the aim of recovering complex and precise movements by decoding motor information in motor related brain areas $[10,11]$. Naturally, such researches have raised the hopes of paralyzed people.

Due to the advances of science and medical technologies, life expectancy has increased. As the person's age increases, so does the development of multiple chronic conditions increases. The number of motor-disabled and/or solitary aged people also increases. However, the resources needed to care for the aged is not meeting the demands. A virtual reality linked to a general purpose BCI could be an alternative for the shortcoming resources on the arrival of aging society and the need of assistive technology.

In this study, we developed a neuron based internet game series called RaviDuel driven by a BCI system as one of the implementations of a virtual reality linked to a general-purpose BCI system. In the implementation, the BCI system developed in our previous study was used [12]. RaviDuel consisted of a betting game RaviDuel $^{+}$and a competitive game RaviDuel ${ }^{*}$. In RaviDuel $^{+}$, players were supposed to bet on the time taken in completing a mission or on one of the subjects that would compete with each other for completing a mission. The mission was a Target-to-Goal (TG) task driven by the BCI system. RaviDuel ${ }^{\#}$ was a player vs. player hitting game, where one of the players was supposed to be a user (a rat, a pet, or a paralyzed person) of the BCI system. The BCI system coded a series of motor functions into prefrontal cortex (PFC) or hippocampal (CA1) region of the brain of the user, generated real-time command signals, and controlled game objects of RaviDuel ${ }^{+}$or RaviDuel ${ }^{\#}$.

\section{System}


Figure 1 shows the schematic diagram of the presented system. The system consists of two parts, the BCI and the internet game systems. The activities of two out of $m$ recorded single neuronal units $s_{j}$ $(j=1,2, \cdots, m)$ were used in real-time in the BCI system to control two-dimensional movements of a water disk or game objects. Combinations of the two

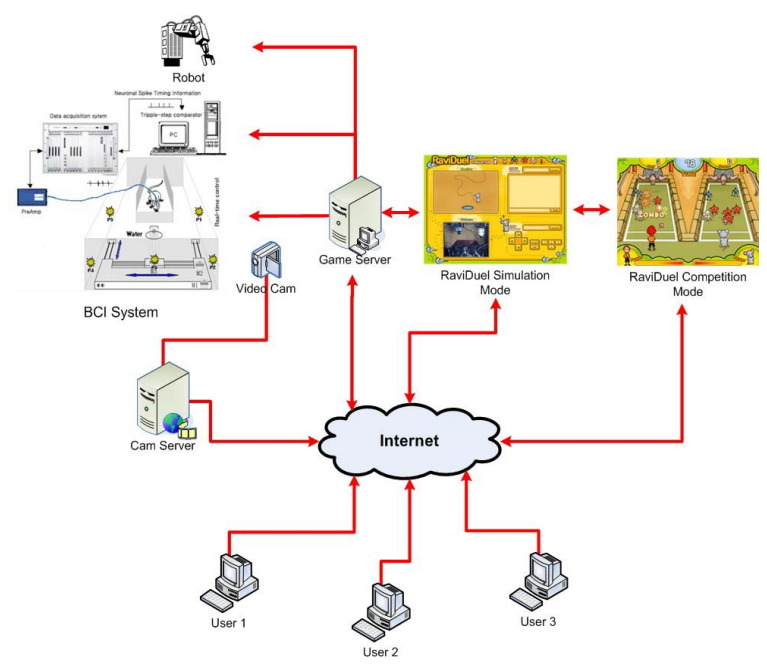

Figure 1. Schematic diagram of the system

neuronal units were mapped into motor function of selecting a direction (left, right, forward, or backward) and motor function of selecting a step count (one, two, three, or zero step count). The firing rate of each of the two neuronal units was quantified and coded into four levels to estimate the relative neuronal activity difference and generate a corresponding command signal for the selected direction and step count. The game system was constructed for game objects to be driven by the command signal output of the BCI system.

\subsection{The BCI system}

Figure 2 shows a block diagram of the BCI system developed in our previous study. The BCI system was composed of data acquisition, feature extraction, source selection, coding, and control units. In the data acquisition unit, neuronal signals recorded from PFC, or CA1 region of the rat brain were amplified, filtered, sorted, and transformed into $m$ spike trains $s_{j}$, $j=1,2, \cdots, m$ in real-time, where $s_{j}=\left(t_{1}^{j}, t_{2}^{j}, \cdots, t_{p}^{j}, \cdots\right)$ and $t_{p}^{j}$ denotes the time of occurrence of the p'th spike emitted by the $j$ 'th neuron. Each spike train during a time interval $\left(\begin{array}{ll}0 & T\end{array}\right]$ was transformed into time series data $X_{j}=\left(x_{1}^{j}, \cdots x_{i}^{j}, \cdots, x_{z}^{j}\right)$ in the feature extraction unit, where $x_{i}^{j}=\rho^{j}\left(t_{i}\right)-\rho^{j}\left(t_{i-1}\right)$ and $z=T / \Delta t \quad$ and $\Delta t=t_{i}-t_{i-1}$ is the bin size of the time series data. The neuronal response function $\rho^{j}\left(t_{i}\right)$ was evaluated as sums over spikes from $\mathrm{j}$ 'th neuron for $0 \leq t \leq i \Delta t$ [13]. The correlation coefficients $r_{j k}$ and the partial correlation coefficients $r_{j k, l}$ of the time series data were then calculated using the equations given in reference [14]. The correlation coefficient $r_{j k}$ measures the correlation between the time series data $X_{j}$ and $X_{k}$. The partial-correlation coefficient $r_{j k, l}$ measures the net correlation between the time series data $X_{j}$ and $X_{k}$ after excluding the common influence of (i.e., holding constant) the time series data $X_{l}$. The source selection unit classified the time series data $X_{j}$ into two groups, correlated, and uncorrelated groups, according to the values of the correlation coefficients. Each group was again subdivided into two subgroups based on the values of the partial correlation

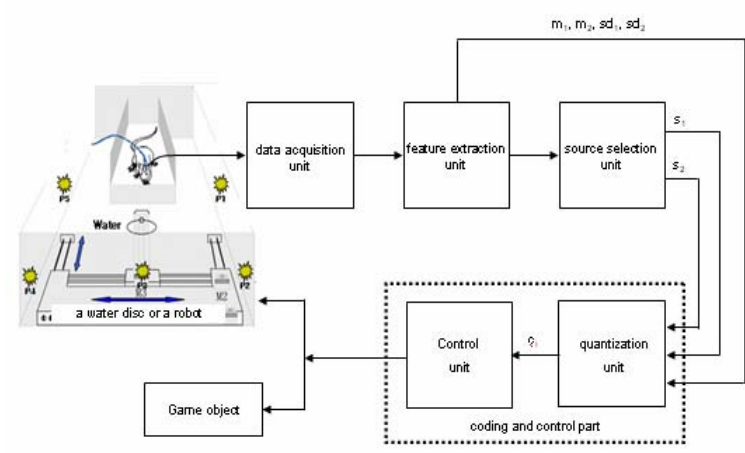

Figure 2. A block diagram of the $\mathrm{BCl}$ system developed in our previous study

coefficients of its elements. Two spike-trains $s_{j_{1}}$ and $s_{j_{2}}$ were then selected, where the corresponding time series data $X_{j_{1}}$ and $X_{j_{2}}$ were belong to the uncorrelated group but not in the same subgroup. In result, $s_{j_{1}}$ and $s_{j_{2}}$ were independent each other as well as had large difference in their correlations with other spike trains $s_{j \neq j_{1}, j_{2}}$. The coding unit coded a series of motor functions into the spike train $s_{j_{1}}$ and $s_{j_{2}}$ by an coding function $f\left(s_{j_{1}}, s_{j_{2}}\right)$ and transformed in real-time the relative difference between the neuronal activities of the spike trains $s_{j_{1}}$ and $s_{j_{2}}$ 
into a command signal corresponding to one of the motor functions. The control unit received the command signal from the coding unit and executed it correspondingly to control the two-dimensional movement of a water disk in the BCI system and game objects of the game system.

\subsection{The game system}

The internet game system was constructed to be driven by the command signal output of the BCI system. Figure 3 shows the flowchart of procedures of a game session in the internet game system. Once a series of initialization steps, such as sign up, login, option selection, etc., is set up, a cam server, a game

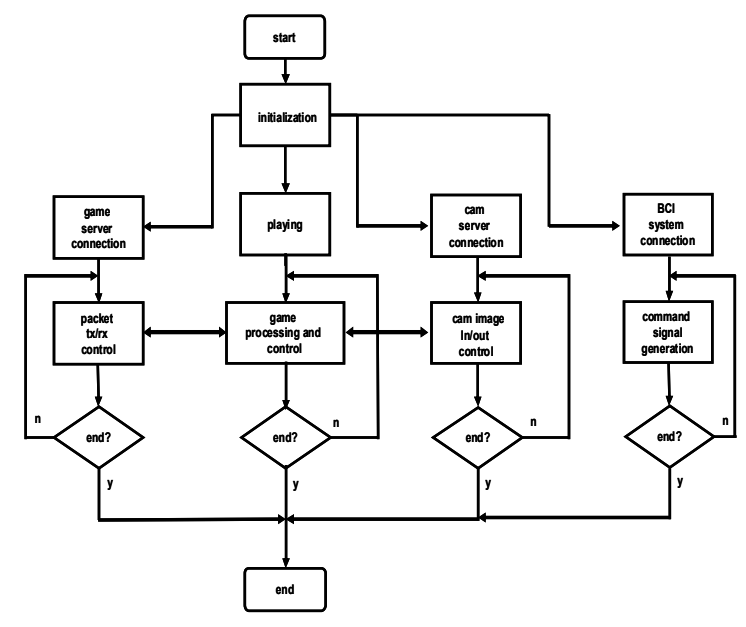

Figure 3. Flowchart of procedures of a game session in the internet game system.

server, and the BCI system are connected to the user system, and a game session starts. The cam server controls and transfers to a game client system real time images captured from a video camera recording the two-dimensional movements of a water disk or a robot and the behavior of a user in the BCI system. The game server controls game procedures and network packet transmissions until a session of a game ends. The game server controls a betting, a competitive, or an interactive game.

\section{Neuron based internet game: RaviDuel}

A betting internet game, RaviDuel ${ }^{+}$, was programmed and simulated. In RaviDuel ${ }^{+}$players bet on the time taken in completing a mission or on one of the subjects who competes with each other for completing a mission. The mission was animated like that a rat finds a water spring, which is linked to a target-to-goal (TG) task in the BCI system. The player was able to preset the position of the water spring. A TG task was for a user of the BCI system to move a water disc from a target position to a goal position in two-dimensional space by using its neuronal activities. The rat was the animated character of the user; the water spring was the animation of the water disc. Initial positions of the rat and the water spring were mapped into the target position and the goal position, respectively. According to a relative difference of the neuronal activities of the user, a command signal was generated for a combination of the directions and the moving speeds during the TG task. The cam server transferred real-time images of the two-dimensional movement of a water disk of the BCI system to the game client system so that the real image and the animated motion could be paralleled. Figure 4 shows a simulation window of the game, RaviDuel ${ }^{+}$. The upperleft part of the window shows the animated motion and the right-lower part, the real image.

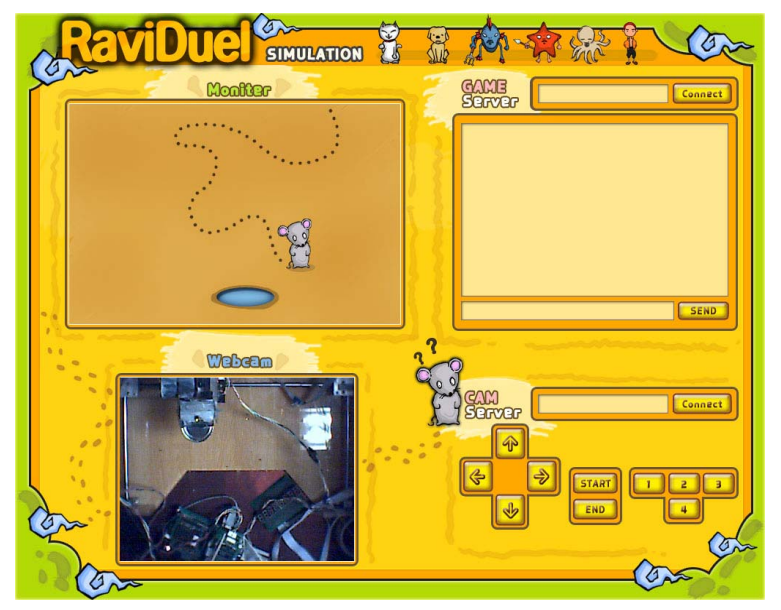

Figure 4. A simulation window of the game, RaviDuel'.

\section{Neuron based internet game: RaviDuel}

A competitive internet game, RaviDuel, was programmed and tested. RaviDuel ${ }^{\#}$ is a player vs. player hitting game, where one of the players was supposed to be a user (a rat, a pet, or a paralyzed person) of the BCI system. Each player had a bowling ball (or a bomb) at the beginning of each game. The player continually encountered new randomly moving animated opponents throughout a session of the game until she/he/it lost. The player got a designated score if she/he/it hit one of the opponents with the bowling ball. 
The player could adjust the speed and the direction of the bowling ball (or a bomb) by using the mouse of his/her system while the user of the BCI system, by using its relative neuronal activity differences and generating a command signal for a combination of the directions and the speeds. The player lost when he/she/it was hit by the opponents or got lower scores than other player within a session of the game. The game was over when either player lost. Figure 5 shows a window of a session of the game, RaviDuel ${ }^{\#}$.

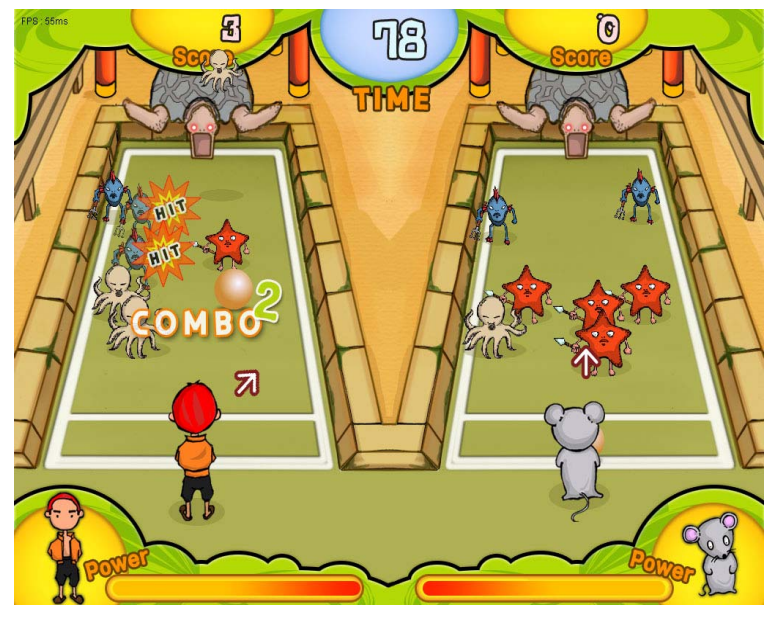

Figure 5. A window of a session of the game, RaviDuel".

\section{Target-to-goal task}

Controlling of a water disc and game objects in twodimensional space was accomplished through a targetto-goal (TG) task using a rat. The subject controlled the direction and the step count of a water disc by using the neuronal activities of $\mathrm{PFC}$ or $\mathrm{CA} 1$ region of its brain for a reward of water in the TG task. The water was contained in a quarter of a circular dish positioned on the top of the water disc. An animal preparation for using in the TG task was approved by the Hallym University Animal Care and Use Committee. Adult male or female Sprague-Dawley rat weighing 200-220g was used. Two multi-wire recording electrodes arrays (8 channels for each array, tungsten microwire, A-M systems, USA, $75 \mu \mathrm{m}$ diameter, teflon-coated) were implanted bilaterally into PFC or CA1 region of both hemispheres of the rat. Four weeks after implantations, the rat was deprived of water for $24 \mathrm{hrs}$. It was then placed in the two-dimensional task space to perform a TG task.

The TG task was to move the water disk from one of five target positions to a predefined goal position for a reward of water. The target position was set to be in a limited area $-6 \leq x \leq+6$ and $-9 \leq y \leq 0$. The coordinates of the target positions were $P_{1}(6,0), P_{2}(6,-9), P_{3}(0,-9), P_{4}(-6,-9)$ and $P_{5}(-6,0)$. The origin of the coordinate $P_{0}(0,0)$ was set up for the predefined goal position.

\section{Conclusions}

A neuron based internet game series called RaviDuel driven by a BCI system was developed as one of the implementations of a virtual reality linked to a general-purpose BCI system. Activities of two single neuronal units recorded from $\mathrm{PFC}$ or CA1 region of SD rats were used in real time to control two-dimensional movements of a game object. A betting game, called RaviDuel $^{+}$, and a competitive game, called RaviDuel were programmed and tested. RaviDuel ${ }^{+}$is a betting game, where players bet on the time taken in completing a mission. RaviDuel ${ }^{\#}$ is a player vs. player hitting game, where one of the players is supposed to be a BCI user (a rat, a robot, or a pet). The constructed game system linked to the BCI system was successfully implemented and the games, RaviDuel ${ }^{+}$and RaviDuel ${ }^{\#}$, were operated in real-time. The current development of new online communication system between animal (or paralyzed) and human showed a promising vision in the fusion technology among neuroscience, robotics and information technologies.

\section{Acknowledgements}

This study was supported by grants to HC SHIN (M103KV010019-06K2201-01910, R11-2000-07501003-0 (2006), Hallym RIC) and by the Korea Research Foundation Grant funded by KRF-2006-531H00003.

\section{References}

[1] Farwell LA and Donchin E., "Talking off the top of your head: toward a mental prosthesis utilizing event-related brain potentials," Electroencephalogr Clin Neurophysiol 1988; vol. 70 , pp. 510-523.

[2] Wolpaw JR, McFarland DJ, Neat GW and Forneris CA., "An EEG-based brain-computer interface for cursor control," Electroencephalogr Clin Neurophysiol 1991, vol. 78, pp. 252-259.

[3] Pfurtscheller G, Flotzinger D and Kalcher J., "BrainComputer Interface - a new communication device for handicapped persons," J Microcomputer Appl 1993, vol.16, pp. 293-299. 
[4] McFarland DJ, Neat GW, Read RF and Wolpaw JR., "An EEG-based method for graded cursor control," Psychobiology 1993, vol. 21, pp. 77-81.

[5] Sutter EE., "The brain response interface: communication through visually induced electrical brain response," $J$ Microcomputer Appl 1992, vol. 15, pp. 31-45.

[6] Paul L. Nunez, "Toward a quantitative description of large-scale neocortical dynamic function and EEG," Behavioral and Brain Sciences 2000, vol. 23(03), pp. 371398.

[7] J. E. Huggins, S. P. Levine, J. A. Fessler, W. M. Sowers, G. Pfurtscheller, B. Graimann, A. Schloegl, D. N. Minecan, R. K. Kushwaha, S. L. BeMent, O. Sagher, L. A. Schuh, "Electrocorticogram as the Basis for a Direct Brain Interface: Opportunities for Improved Detection Accuracy," Proceedings of the $1^{\text {st }}$ International IEEE EMBS Conference on Neural Engineering, Capri Island March 2003, pp. 20-22.

[8] Mijail D. Serruya, Nicholas G. Hatsopoulos, Liam Paninski, Matthew R. Fellows,John P. Donoghue, "Instant neural control of a movement signal," Nature 2002, vol. 416, pp. 141-142.

[9] Jhon K. Chapin, "Using multi-neuron population recordings for neural prosthetics," Nature Neurosci 2004, vol. 7, pp. 452-455.

[10] Chapin, J.K., Moxon, K.A., Markowitz, R.S., and Nicolelis, M.A.L., "Real-time control of a robot arm using simultaneously recorded neurons in the motor cortex," Nature Neurosci., 1999, vol. 2 pp. 664-670.

[11] Wu, W., Black, M.J., Mumford, D., Gao, Y., Bienenstock, E., Donoghue, J. P., "Modeling and decoding motor cortical activity using a switching Kalman filter," IEEE Transactions on Biomedical Engineering 2004, vol. 51(6), pp. 933-942.

[12] U. Lee, H-J. Lee, S. Kim and H.-C. Shin, "Development of Intracranial brain-computer interface system using nonmotor brain area for series of motor functions," Electronics Letters 2006, vol. 42(4), pp. 198-200.

[13] P Dayan and L F Abbott, "Theoretical Neuroscience: Computational and Mathematical Modeling of Neural System,”. MIT Press, 2001.

[14] Richard Izzett, "SPSS Windows Instructions for PSYCH. 280 \& PSYCH. 290," $2^{\text {nd }}$ edition, http://www.oswego.edu/ psychol/spss/partial.pdf. 